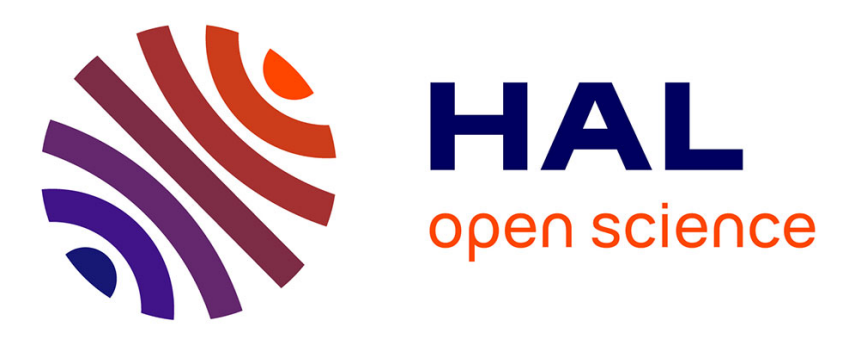

\title{
High Resolution Hippocampus Subfield Segmentation Using Multispectral Multiatlas Patch-Based Label Fusion
}

\author{
José V Romero, Pierrick Coupe, José V Manjón
}

\section{- To cite this version:}

José V Romero, Pierrick Coupe, José V Manjón. High Resolution Hippocampus Subfield Segmentation Using Multispectral Multiatlas Patch-Based Label Fusion. Patch-Based Techniques in Medical Imaging (MICCAI), Oct 2016, Athènes, Greece. pp.117 - 124, 10.1007/978-3-319-47118-1_15 . hal01398769

\author{
HAL Id: hal-01398769 \\ https://hal.science/hal-01398769
}

Submitted on 17 Nov 2016

HAL is a multi-disciplinary open access archive for the deposit and dissemination of scientific research documents, whether they are published or not. The documents may come from teaching and research institutions in France or abroad, or from public or private research centers.
L'archive ouverte pluridisciplinaire HAL, est destinée au dépôt et à la diffusion de documents scientifiques de niveau recherche, publiés ou non, émanant des établissements d'enseignement et de recherche français ou étrangers, des laboratoires publics ou privés. 


\title{
High resolution hippocampus subfield segmentation using multispectral multiatlas patch-based label fusion
}

\author{
José E. Romero ${ }^{1}$, Pierrick Coupe ${ }^{2,3}$, José V. Manjón ${ }^{1}$ \\ ${ }^{1}$ Instituto de Aplicaciones de las Tecnologías de la Información y de las Comunicaciones \\ Avanzadas (ITACA), Universitat Politècnica de València, Camino de Vera s/n, 46022 \\ Valencia, España. \\ ${ }^{2}$ Univ. Bordeaux, LaBRI, UMR 5800, PICTURA, F-33400 Talence, France. \\ ${ }^{3}$ CNRS, LaBRI, UMR 5800, PICTURA, F-33400 Talence, France.
}

\begin{abstract}
The hippocampus is a brain structure that is involved in several cognitive functions such as memory and learning. It is a structure of grate interest due to its relationship to neurodegenerative processes such as the Alzheimer's disease. In this work, we propose a novel multispectral multiatlas patch-based method to automatically segment hippocampus subfields using high resolution T1-weighted and T2-weighted magnetic resonance images (MRI). The proposed method works well also on standard resolution images after superresolution and consistently performs better than monospectral version. Finally, the proposed method was compared with similar state-of-the-art methods showing better results in terms of both accuracy and efficiency.
\end{abstract}

\section{Introduction}

The hippocampus (HC) is a complex gray matter structure of the brain located under the surface of each temporal lobe. It is involved in many cognitive functions such as memory and spatial reasoning [1]. It presents changes in its structure across the lifespan related to normal aging [2] as well as to several dysfunctions like epilepsy [3], schizophrenia [4] and Alzheimer's disease [5].

The HC is a three dimensional curved structure that has been linked to the sea horse. The hippocampus is composed of multiple subfields that can be divided into sections called the dentate gyrus, the cornu ammonis (CA) and the subiculum. The $\mathrm{CA}$ is also subdivided in sub-sections CA1, CA2, CA3, CA4, layers alveus, stratum oriens, stratum pyramidale, stratum radiatum, stratum lancosum and stratum moleculare. These layers present a high neuron density and are very compact so high resolution imaging is required to identify them.

Due to this morphological complexity and limitations of MR image resolution, most of past studies have been performed over the whole hippocampus volume by segmenting it as a single object [6]. These studies showed that the whole hippocampus volume is a good biomarker for Alzheimer's disease [7]. However, hippocampus subfields have shown to be affected differently by AD and normal aging in ex-vivo studies [5]. 
Although high resolution MRI is becoming more accessible in certain scenarios, these data have been traditionally manually segmented. However, manual segmentation is a highly time consuming procedure which requires trained raters specially for complex structures such as hippocampus subfields. Taking about 50 hours per case it is impossible to apply manual delineation to large cohort studies.

To avoid this problem automated solutions have been developed in the last years. The method proposed by Chakravarty et al. consists of a multiatlas method based on the estimation of several non-linear deformations and a label fusion step [8]. Also using a multiatlas approach Yushkevich et al. proposed a method where a multiatlas approach is combined with a similarity-weighted voting and a learning-based label bias correction [9]. In a different manner, Van Leemput et al. used a statistical model of MR image formation around the hippocampus to produce automatic segmentation [10]. Recently, Iglesias et al. pursued this work and replaced the model by a more accurate atlas generated using ultra-high resolution ex-vivo MR images [11].

In this work we propose a fast and accurate multispectral multiatlas patch-based method to segment the hippocampus subfields according to the atlas presented in [12]. The proposed method is an extension of a recently proposed segmentation algorithm called OPAL [13]. This extension integrates multispectral similarity estimation and a novel non-local regularization post-processing step.

\section{Material and Methods}

\section{$2.1 \quad$ Image data}

In this paper, we used a High Resolution (HR) dataset composed of 5 cases with T1weighted and $\mathrm{T} 2$-weighted images to construct a library of manually labeled cases. The HR images are publicly available at the CoBrALab website (http://cobralab.ca/atlases). Both, the HR images used as input and the manually labeled validation dataset are the same as those used in Pipitone et al. [14].

To create the HR atlases MR images were taken from 5 healthy volunteers (2 males, 3 females, aged 29-57). T1- and T2-weighted images were acquired for all subjects on a 3 T GE Discovery MR 750 system (General Electric, Milwaukee, WI) using an 8-channel head coil. High-resolution T1-weighted images were acquired using the 3D inversion-prepared fast spoiled gradient-recalled echo acquisition, FSPGR-BRAVO, in a scan time of $\sim 20$ min, with the following parameters: $\mathrm{TE} / \mathrm{TR}=4.3 \mathrm{~ms} / 9.2 \mathrm{~ms}, \mathrm{TI}=650 \mathrm{~ms}, \alpha=8^{\circ}, 2-\mathrm{NEX}, \mathrm{FOV}=22 \mathrm{~cm}$, slice thickness $=0.6$ $\mathrm{mm}, 384 \times 384$ in-plane steps for an approximately isotropic resolution of $0.6 \mathrm{~mm}$ dimension voxels. High-resolution T2-weighted images were acquired using the $3 \mathrm{D}$ fast spin echo acquisition, FSE-CUBE, in a scan time of $\sim 16$ min, with the following parameters: TE/TR $=95.3 \mathrm{~ms} / 2500 \mathrm{~ms}, \mathrm{ETL}=100 \mathrm{~ms}, 2 \mathrm{NEX}, \mathrm{FOV}=22 \mathrm{~cm}$, slice thickness $=0.6 \mathrm{~mm}, 384 \times 384$ in-plane steps for approximately isotropic $0.6 \mathrm{~mm}$ dimension voxels. Reconstruction filters, ZIPX2 and ZIP512, were also used resulting in a final isotropic $0.3 \mathrm{~mm}$ dimension voxels. All 2-NEX scans were then repeated three times and averaged for a total of 6-NEX. The hippocampi and each of their subfields were 
segmented manually by an expert rater including 5 labels (CA1, CA2/3, CA4/dentate gyrus, stratum radiatum/stratum lacunosum/stratum moleculare (SR/SL/SM), and subiculum). For more details about the labeling protocol please read the original paper [12].

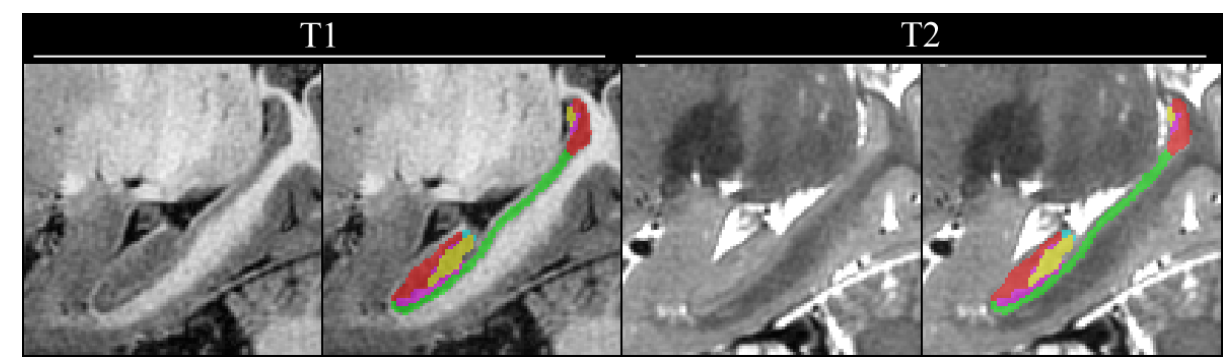

Figure 1: Example of an HR MRI case. Figure shows T1w and T2w images and its corresponding manual segmentation.

\subsection{Preprocessing}

All the images (T1 and T2) were first filtered with a spatially adaptive non-local means filter [20] and inhomogeneity corrected using the N4 method [21]. Later, they were linearly registered to the Montreal Neurological Institute space (MNI) using the ANTS package [15] and the MNI152 template. Next, we left-right flipped the images and cropped them to the right hippocampus area so we have 10 right hippocampus crops. Note that after considering the flipped versions of the images only one of both hippocampi has to be considered otherwise we would have the same hippocampi twice. After that, we non-linearly registered the cropped images to the cropped MNI152 template to better match the hippocampus anatomy. Finally, we normalized the images to have the same mean and standard deviation as the MNI152 template and a sharpening operation (by substracting the laplacian of the image) was applied to the images to minimize the blurring introduced by the interpolation during the nonlinear registration process.

\subsection{Library}

Multiatlas based segmentation methods are based on the use of a library of manually labeled cases. In our case, to construct the library, we processed the $5 \mathrm{HR}$ images (T1 and T2 versions) as described in the previous section to finally have a 10 hippocampi library.

\subsection{Labeling and regularization}

Multispectral Optimized PatchMatch (MOPAL) 
One of the most time consuming parts of non-local label fusion technique is the patch matching step. To reduce the computational burden of this process, we used an adaptation of the OPAL method [13] that is a 3D adaptation of the patchmatch technique proposed by Barnes et al. [16].. For more details, see the original OPAL paper [13]

In the original OPAL method, the probability maps from 2 different scales (patch sizes) were mixed using a late fusion scheme with equal weights for both scales. In this work, we learn a label dependent mixing coefficient to balance the different scale contributions per label using a gradient descend technique. Moreover, the maps returned by OPAL consists of a probability map for each label that is being considered. These maps are the processed to obtain the final segmentation by choosing the label with maximum probability for each voxel. When using a multiscale approach two probability maps are obtained for each label (one for patch size). Therefore a combination is required to generate a single probability map for each label. This step was done using a global mixing coefficient giving equal weight to every label. Given that different labels have different subjacent anatomy it is possible that different structures perform obtain better segmentation from one benefit more from one scale than the other so we calculated an individual mixing coefficient for every label to maximize the segmentation accuracy. We use multispectral distance computation taking into account information derived from T1 and T2 MRI in order to compute patch correspondences in a more robust manner. OPAL estimates the quality of a match by computing a distance as the sum of squared differences (SSD). This proposed multispectral distance in a balanced sum of SSDs (one per channel) that we called multispectral sum of squared differences (MSSD):

$$
M S S D=\frac{1}{M}\left((1-\lambda)\left\|P\left(A_{i}\right)-P\left(A_{s, j}^{\prime}\right)\right\|_{2}^{2}+\lambda\left\|P\left(B_{i}^{\prime}\right)-P\left(B_{s, j}^{\prime}\right)\right\|_{2}^{2}\right)
$$

Where $A$ and $B$ represent the target image for T1 and T2, $A$ ' and $B$ ' represent the libraries for T1 and T2 repectively, $P\left(A_{i}\right) \in A$ is a patch form image $A$ centered on the coordinates $i, P\left(B_{j}\right) \in B$ is a patch from image $B$ centered on the coordinates $j, \lambda$ is a coefficient required to balance the different distance contributions and $M$ is the number of voxels per patch.

\section{Label regularization}

Automatic segmentations produced by MOPAL are performed at patch. Even thou patchwise segmentation implies regularization it is not sufficient to produce smooth contours.. Since hippocampus subfields are regular and layered structures, some extra regularization may help to produce feasible segmentations. To this end, the probability map (resulting from the combination of both considered scales) is regularized using a non-local means filter [17]. The final segmentation is generated by calculation the maximum probability for each voxel for all 5 structures probability maps. 


\section{$3 \quad$ Experiments and results}

In this section a set of experiments are presented to show the performance of the method and the effect of the proposed modifications. All the experiments have been done by processing the cases from the library described before in a leave-two-out fashion by removing the case being processed and its mirror counterpart (thus using only a library of 8 images instead of 10).

\subsection{MOPAL parameters}

OPAL [13] was developed and evaluated for the segmentation of the whole hippocampus, so an optimization of the method parameters was performed. To measure the accuracy we used the DICE [18] coefficient for all the 5 structures. In all the experiments, we set the patch sizes to $3 \times 3 \times 3$ and $9 \times 9 \times 9$ voxels, for each scale respectively. The restricted search area was set to $7 \times 7 \times 7$ voxels. The number of independent Patch Matches was set to 32 and the number of iterations of OPAL to 4 . The 6 scale mixing coefficients (5 structures + background) ( $\mathrm{alfa}=[0.4711,0.3443,0.3826,0.3900$, $0.8439,0.7715])$ and MSSD balance parameters $(\lambda=0.9)$ were empirically estimated.

The multispectral proposed method was compared with the corresponding monomodal version (for both T1 and T2 MRI) using the same parameters with exception of $\lambda$. In Table 1, it can be observed how the T2 based results are better than the corresponding $\mathrm{T} 1$ based results. This result is in line with results of previous studies on this topic (ref??) . However, T2 based segmentation seems to be worse than T1 based for CA2/CA3 structure while multispectral based segmentation benefits from both $\mathrm{T} 1$ and $\mathrm{T} 2$ based segmentation and performs well for all the structures.

Table 1: Mean DICE and standard deviation for each structure segmentation using high resolution T1, T2 and Multispectral respectively. Best results in bold. Significant differences between $T 1$ and $T 1+T 2$ are marked with * and between $T 2$ and $T 1+T 2$ are marked with $\uparrow(p<0.05)$.

\begin{tabular}{cccc}
\hline Structure & T1 HR & T2 HR & T1+T2 HR \\
\hline Average & $0.6253 \pm 0.0926$ & $0.6762 \pm 0.0716$ & $\mathbf{0 . 6 9 5 7} \pm 0.0651^{*}$ \\
\hline CA 1 & $0.6752 \pm 0.0254$ & $0.7304 \pm 0.0464$ & $\mathbf{0 . 7 4 3 9} \pm 0.0298^{*}$ \\
CA $\mid C A 3$ & $0.6865 \pm 0.0367$ & $0.6468 \pm 0.0642$ & $\mathbf{0 . 7 0 1 5} \pm 0.0398 \dagger$ \\
$C A 4 \mid D G$ & $0.7129 \pm 0.0319$ & $0.7709 \pm 0.0323$ & $\mathbf{0 . 7 7 1 0} \pm 0.0255^{*}$ \\
SR $|S L| S M$ & $0.5085 \pm 0.0960$ & $0.5994 \pm 0.0531$ & $\mathbf{0 . 6 1 4 5} \pm 0.0632^{*}$ \\
Subiculum & $0.5434 \pm 0.0473$ & $0.6336 \pm 0.0485$ & $\mathbf{0 . 6 4 7 6} \pm 0.0406^{*}$ \\
\hline Hippocampus & $0.8782 \pm 0.0174$ & $0.8880 \pm 0.0171$ & $\mathbf{0 . 9 0 1 1} \pm 0.0097$ \\
\hline
\end{tabular}

\subsection{Label regularization}

We performed also an experiment to measure the effect of the label regularization on the segmentation results. We optimized the non-local means filter parameters (patch size $=3 \times 3 \times 3$, search volume $=7 \times 7 \times 7$ and the smoothing parameter $h=0.02$ ). In 
Table 2 improvements can be seen in almost every structure compared to Table 1.In Figure 2 an example of the segmentation results is presented.

Table2: Mean DICE and standard deviation for each structure segmentation using high resolution T1, T2 and Multispectral respectively. Values showing improvement with the regularization in bold.

\begin{tabular}{cccc}
\hline Structure & $\boldsymbol{T 1}$ HR & $\boldsymbol{T 2}$ HR & $\boldsymbol{T 1}+\boldsymbol{T} 2$ HR \\
\hline Average & $\mathbf{0 . 6 2 8 6} \pm 0.0930$ & $\mathbf{0 . 6 7 7 5} \pm 0.0704$ & $\mathbf{0 . 6 9 8 5} \pm 0.0657$ \\
\hline$C A 1$ & $\mathbf{0 . 6 7 8 8} \pm 0.0252$ & $\mathbf{0 . 7 3 1 4} \pm 0.0477$ & $\mathbf{0 . 7 4 8 7} \pm 0.0287$ \\
$C A 2 \mid C A 3$ & $\mathbf{0 . 6 9 0 1} \pm 0.0372$ & $\mathbf{0 . 6 4 9 1} \pm 0.0638$ & $\mathbf{0 . 7 0 5 8} \pm 0.0381$ \\
$C A 4 \mid D G$ & $\mathbf{0 . 7 1 6 4} \pm 0.0319$ & $0.7705 \pm 0.0330$ & $\mathbf{0 . 7 7 3 0} \pm 0.0257$ \\
SR $|S L| S M$ & $\mathbf{0 . 5 1 0 2} \pm 0.0971$ & $\mathbf{0 . 6 0 3 2} \pm 0.0558$ & $\mathbf{0 . 6 1 7 6} \pm 0.0653$ \\
Subiculum & $\mathbf{0 . 5 4 7 6} \pm 0.0483$ & $0.6332 \pm 0.0488$ & $0.6473 \pm 0.0436$ \\
\hline Hippocampus & $\mathbf{0 . 8 8 0 6} \pm 0.0178$ & $\mathbf{0 . 8 8 9 0} \pm 0.0172$ & $\mathbf{0 . 9 0 3 2} \pm 0.0104$ \\
\hline
\end{tabular}

\subsection{Standard resolution vs High resolution}

As high resolution MR images are not widely available, especially in clinical environments, we analyzed how the proposed method performs on standard resolution images. For this purpose, we reduced de resolution of the HR images by a factor 2 by convolving the HR images with a $2 \times 2 \times 2$ boxcar kernel and then decimating the resulting image by a factor 2 . As the library used in our method is located in the $0.5 \mathrm{~mm}$ resolution MNI space, the obtained down-sampled images were upsampled by a factor 2 using BSpline interpolation and a superresolution method called Local Adaptive SR (LASR) [19]. Results are shown in Table 3. As can be noticed, segmentations performed on images up-sampled with SR were better than using interpolation. Moreover, this experiment shows that the proposed method is able to produce competitive results when using standard resolution images.

Table 3: Mean DICE and standard deviation for each structure segmentation using the high resolution library and applying BSpline interpolation and LASR to the previously downsampled image to be segmented. Segmentation produced using the multispectral version of the method. Best results in bold.

\begin{tabular}{ccc}
\hline Structure & BSpline & LASR \\
\hline Average & $0.6696 \pm 0.0738$ & $\mathbf{0 . 6 8 8 4} \pm \mathbf{0 . 0 6 8 2}$ \\
\hline$C A 1$ & $0.7247 \pm 0.0382$ & $\mathbf{0 . 7 4 2 0} \pm \mathbf{0 . 0 2 8 6}$ \\
$C A 2 \mid C A 3$ & $0.6878 \pm 0.0516$ & $\mathbf{0 . 7 0 1 0} \pm \mathbf{0 . 0 4 3 7}$ \\
$C A 4 \mid D G$ & $0.7498 \pm 0.0358$ & $\mathbf{0 . 7 6 2 2} \pm \mathbf{0 . 0 2 9 1}$ \\
SR $|S L| S M$ & $0.5834 \pm 0.0688$ & $\mathbf{0 . 6 0 6 0} \pm \mathbf{0 . 0 6 5 6}$ \\
Subiculum & $0.6023 \pm 0.0495$ & $\mathbf{0 . 6 3 0 8} \pm \mathbf{0 . 0 4 4 2}$ \\
\hline Hippocampus & $0.9001 \pm 0.0102$ & $\mathbf{0 . 9 0 4 2} \pm \mathbf{0 . 0 0 9 5}$ \\
\hline
\end{tabular}




\subsection{Comparison}

We compared our method with another recent method applied to hippocampus segmentation using the same number of structures and labeling protocol. The compared method is called MAGeT [8] and is lies on the estimation of a large number of non-linear deformations followed by a majority vote label fusion. Table 4 shows that the proposed method obtained higher DICE coefficients for all the structures. In terms of computation efficiency our method requires only a few minutes while MAGeT has an execution time of several ours per case. It has to be noted that MAGET results are computed in MNI space at $0.9 \mathrm{~mm}$ resolution while MOPAL results are computed at $0.5 \mathrm{~mm}$ resolution.

Table 4: Mean DICE for each structure. Segmentation performed by MAGeT and the proposed method. Best results in bold.

\begin{tabular}{ccc}
\hline Structure & MAGET(0.9mm) & Proposed (0.5mm) \\
\hline Average & 0.526 & $\mathbf{0 . 6 9 8 5}$ \\
\hline$C A 1$ & 0.563 & $\mathbf{0 . 7 4 8 7}$ \\
$C A 2 \mid C A 3$ & 0.412 & $\mathbf{0 . 7 0 5 8}$ \\
$C A 4 \mid D G$ & 0.647 & $\mathbf{0 . 7 7 3 0}$ \\
$S R|S L| S M$ & 0.428 & $\mathbf{0 . 6 1 7 6}$ \\
Subiculum & 0.58 & $\mathbf{0 . 6 4 7 3}$ \\
\hline Hippocampus & 0.816 & $\mathbf{0 . 9 0 3 2}$ \\
\hline
\end{tabular}

\section{Discussion}

In this paper we present a new hippocampus subfield segmentation method based on an extension of a recent method called OPAL. The proposed method achieves better segmentation results using an improved multiscale mixing strategy and especially a novel multispectral distance computation that enables to find better matches. Also, a post-processing step has been also added to regularize label probability maps

The proposed method has been showed to perform well on standard resolution images, obtaining competitive on typical clinical data. This fact is of special importance because it will allow analyzing a large number of retrospective data. Finally, it has been shown that the proposed method compares well to another related state-of-art method obtaining better results in terms of both accuracy and reduced execution time.

\section{Acknowledgements}

This research was supported by the Spanish grant TIN2013-43457-R from the Ministerio de Economia y competitividad. This study has been carried out with financial support from the French State, managed by the French National Research Agency (ANR) in the frame of the Investments for the future Program IdEx Bordeaux (ANR10-IDEX-03-02, HL-MRI Project), Cluster of excellence CPU and TRAIL (HR-DTI ANR-10-LABX-57) and the CNRS multidisciplinary project "Défi imag'In". 


\section{$6 \quad$ References}

1. Milner, B, Psychological defects produced by temporal lobe excision Res. Publ. Assoc. Res. Nerv. Ment. Dis., 36, pp. 244-257, (1958).

2. Petersen, R et al. Memory and MRI-based hippocampal volumes in aging and AD Neurology, 54 (3), pp. 581-587, (2000).

3. Cendes, $\mathrm{F}$ et al., MRI volumetric measurement of amygdala and hippocampus in temporal lobe epilepsy Neurology, 43 (4), pp. 719-725, (1993).

4. Altshuler, LL et al., Amygdala enlargement in bipolar disorder and hippocampal reduction in schizophrenia: an MRI study demonstrating neuroanatomic specificity Arch. Gen. Psychiatry, 55 (7), p. 663, (1998).

5. Braak H and Braak E, Neuropathological stageing of Alzheimer-related changes, Acta Neuropathol., 82 (4), pp. 239-259, (1991)

6. Chupin, $\mathrm{M}$ et al., Fully automatic hippocampus segmentation and classification in Alzheimer's disease and mild cognitive impairment applied on data from ADNI Hippocampus, 19 (6), pp. 579-587, (2009).

7. Jack, $\mathrm{C}$ et al., Prediction of $\mathrm{AD}$ with MRI-based hippocampal volume in mild cognitive impairment Neurology, 52 (7), pp. 1397-1403, (1999).

8. Chakravarty, $M$. et al., Performing label-fusion-based segmentation using multiple automatically generated templates, Human brain mapping,10(34), pp. 2635 - 2654, (2013).

9. Yushkevich, PA et al., Automated volumetry and regional thickness analysis of hippocampal subfields and medial temporal cortical structures in mild cognitive impairment Hum. Brain Mapp., 36 (1), pp. 258-287, (2015).

10. Van Leemput, $\mathrm{K}$ el al., Automated segmentation of hippocampal subfields from ultra-high resolution in vivo MRI Hippocampus, 19 (6), pp. 549-557, (2009).

11. Iglesias JE et al., A computational atlas of the hippocampal formation using ex vivo, ultrahigh resolution MRI: Application to adaptive segmentation of in vivo MRI, NeuroImage, 115 (15), pp. 117-137, (2015).

12. Winterburn JL et al., A novel in vivo atlas of human hippocampal subfields using highresolution 3 T magnetic resonance imaging, NeuroImage, 74, 254-265, (2013).

13. Giraud R et al., An Optimized PatchMatch for Multi-scale and Multi-feature Label Fusion. NeuroImage, 124, pp. 770-782, (2016).

14. Pipitone JL et al., Multi-atlas segmentation of the whole hippocampus and subfields using multiple automatically generated templates, Neuroimage, 101(1), pp. 494-512, (2014).

15. Avants, BB et al., Advanced normalization tools (ANTS), Insight Journal, (2009).

16. Barnes, C, et al., PatchMatch: a randomized correspondence algorithm for structural image editing, ACM Trans Graph, 28(3), (2009).

17. Coupé, P et al., Adaptive Multiresolution Non-Local Means Filter for 3D MR Image Denoising, IET Image processing, 6(5), pp. 558-568, (2012).

18. Zijdenbos AP et al., Morphometric analysis of white matter lesions in MR images: method and validation, IEEE Transactions on Medical Imaging, 13(4), pp. 716-724, (1994)

19. Coupé P et al., Collaborative patch-based super-resolution for diffusion-weighted images, NeuroImage, 83, pp. 245-261, (2013).

20. Manjón, J.V. et al. Adaptive non-local means denoising of MR images with spatially varying noise levels. J Magn Re-son Imaging. 31, 192-203 (2010).

21. Tustison, N.J.et al. N4ITK: improved N3 bias correction. IEEE Trans Med Imaging 29(6): 1310 - 1320. (2010) 